\title{
An Improved Dynamic Load Balancing Routing Protocol Based on Mesh Network
}

\author{
Lu Yan and Ding Xiong \\ Hunan International Economics University, Changsha, China
}

\begin{abstract}
An improved dynamic load balancing routing protocol is proposed based on mesh networks. A cost-effective integrated link state routing algorithm is designed in this protocol, and the mechanism of real-time monitoring and adjustment is adopted in this algorithm for the link state. The source nodes with routing redundancy are notified actively, and the secondary routing is used or focused when the link changes greatly. Moreover, a real-time backoff algorithm is proposed in this protocol. The network fairness problem is solved when the multi-path task and the single-path task compete for the link. The simulation results show that the proposed protocol can effectively avoid the congestion of network nodes. Better dynamic load balancing effect can be achieved, and the utilization of network resources is improved.
\end{abstract}

ACM CCS (2012) Classification: Networks $\rightarrow$ Network protocols $\rightarrow$ Network protocol design

Keywords: load balancing, link-state, multi-path routing, wireless mesh network, metric

\section{Introduction}

Wireless mesh Network (WMN) is a kind of multi-hop, self-organizing and self-healing broadband wireless network structure which will be the ideal networking mode for wireless metropolitan area backbone network. Wireless mesh network core technology is a routing protocol, including three major types: first-type routing protocol, reactive routing protocol and hybrid routing protocol. The first-type routing protocol constantly detects network topology and link quality changes. The routing table is updated according to the change, such as
DSDV [2], WRP [3], [4] and CGSR [5]. Reactive routing is also called on-demand routing, for example, AODV [6], DSR [7], TORA [8]. The node does not need to maintain real-time and accurate routing information, and it's necessary to find the route only when needed. Hybrid routing protocol combines the advantages of both. For example, the ZRP protocol is a hierarchical structure routing protocol with a mix of on-demand and active routing strategy [9]. However, the above standard routing protocols lack effective load balancing mechanism. Some nodes take more tasks (overload), while others are idle (light load), not making full use of WMN resources.

Multi-path routing is a solution to load balancing. The so-called multi-path routing refers to a route discovery process where a number of routes can be found to reach the destination node. All these routes are needed to transmit data, so the utilization of the entire network resources is improved. In recent years, some of the multi-path routing protocols are developed mostly based on DSR or AODV, such as SMR [10], AOMDV [11], MSR [12], AODV-BR [13], NDM [14].

The MSR and SMR, based on DSR, are typical multi-path protocols. MSR, a multi-path extension to the DSR protocol, combines on-demand, multi-path and source routing. The key point of the agreement is to reduce group and send delay, which raises the load balancing ability of the whole network. According to the detection result, the weighted cyclic schedul- 
ing algorithm is used, and the data flow can be divided into multiple independent paths, so that the load balance can be achieved [15]. In this protocol, the delay is used as the metric of the path specification. The state of the path is sensed by RTT, and the purpose is to make full use of network resources. SMR, an on-demand routing protocol, uses a split approach. Data packets are distributed in two special paths: one is the shortest path, and the other is the shortest path with the greatest independence. This method can not only effectively utilize the network resources with the load balancing ability of the whole network improved, but also effectively prevent the network congestion under the condition of large load.

Based on the AODV protocol, AOMDV, AODV$\mathrm{BR}, \mathrm{NDM}, \mathrm{AOMDV}$ (vector routing protocol with on-demand multi-path distance) obtain a number of acyclic and links disjoint path in the route discovery process. AOMDV can take full advantage of the existing routing information in AODV and only needs to add a small amount of additional overhead when calculating multipath. AODV-BR (Backup Routing Protocol) introduces some new ideas in loopback routing reply messages, and maintains multiple routes to the destination node without increasing the number of control messages. The NDM protocol increases the routing request node table, records each intermediate node from the source nodes to the destination node and takes into account the cost of routing and complexity factors. NDM generally chooses the number of paths according to the specific situation.

C. J. Lin et al. proposed a mesh network load balancing scheme based on resource allocation [16]. S. Waharte et al. proposed a load-aware mesh network load balancing scheme [17]. Kharasani et al. proposed a load-balanced routing algorithm for multi-radio wireless mesh networks [18]. A disjoint multi-path routing protocol (MRAODV-DM) was proposed in [19]. Liu et al. proposed a load balancing routing protocol based on ant colony optimization algorithm in wireless mesh network [20]. Hybrid Wireless mesh Routing Protocol HWMP is used in the draft IEEE802.11s. It is a hybrid routing protocol in the mesh Point (MP), which can be used as the root node of the routing tree. The route can be established quickly to the root node, and the best route can be discovered and maintained by the MP itself. HWMP combines both apriori routing and counterfeit routing. Tree-based routing (TBR) [21] and an improved protocol are mainly used for AODV (Radio-Metric AODV, RM-AODV) [22]. HWMP greatly improves the performance of WMN, but it relies too much on the processing of the root node.

A wireless mesh network load balancing protocol NNP-L2MPM was proposed on the basis of neural network prediction model [23]. The protocol calculates the path quality according to the flooded HELLO packet in the network, and selects the optimal next hop to the destination node and uses the MAC layer. The interface queue length is used as the basis for measuring the traffic load. Then, the RBF neural network prediction model is used to predict the node traffic load in the mesh network. The path quality is optimized according to the predicted traffic load at the next moment and the routing update is implemented in advance to avoid the intermediate node. If congestion occurs, it can improve network performance in turn. Based on the sand collapse model and its improvement, Y. C. Zhang proposed a load balancing algorithm for wireless mesh networks, focusing on the trigger conditions of load balancing, candidate node set calculation and load distribution mechanism [24].

\section{Methodology}

DSR adopts the source routing method. The source host knows which path can reach the target host and the proper path can be selected to send the data packet. DSR is used to design the dynamic source routing protocol of multi-path routing load balance (LBDSRM).

\subsection{Load Balancing Metric}

The routing criteria includes hop count, RTT, per-hop packet delay, ETT, ETX, WCETT [25], [26] in the WMN routing protocol. The hop count measures the link quality by counting the number of nodes in the link. RTT (Round Trip Time) measures the link quality by measuring the Round Trip Time of unicast detection packets. Each hop packet pair delay is measured by a node and sent to the neighbor node. The delay between the link states is analyzed between 
two data packets. ETX (expected number of transmissions) estimates the number of retransmissions of unicast packets by counting the loss rate of the neighbor test packets. WCETT (weighted cumulative ETT), a combination of the throughput of the entire path, will be controlled by the impact of the bottleneck channel. In that way, the number of hops is increased based on the consumption of resources, and the weighted average is found.

Although the algorithm is simply based on hop count, there are many shortest paths with the same hop number in a dense network. If only a few routes are selected with some minimum hop criteria, it is highly probable that the optimal link is not selected. If the RTT, per-hop packet delay and ETX algorithm are used as routing standards, although great progress could be made in many ways compared with the use of hops, some extra expenses will be brought to the whole network system, as they collect the state information of the link by sending detect packets. WCETT, based on multi-channel system, can resolve the balance of throughput, bandwidth, hop count and delay, but it still cannot resolve the problem of the cost of collecting information. In the LBDSRM protocol, we design a routing metric with a small cost, so as to improve the running efficiency of the protocol. The metric is computed based on the collection of node information, including bandwidth, amount of data sent, buffer space and other information.

The real-time network card bandwidth of the node can reflect the state of the communication link of the node to a certain extent. Real-time bandwidth of the card $B_{i}$ is defined for the wireless router node $i$. The numbers of received $\left(R_{i}\right)$ and transmitted $\left(S_{i}\right)$ data are recorded at node $i$, and the throughput performance of wireless routing node $i$ is defined as $C_{i}=R_{i}+S_{i}$.

The numbers of data packets will be processed in transmit and receive buffer queues, which can reflect the state of the node link. The congestion index is defined as $S Q_{i}=1$ when the ratio of the sending queue length to the maximum queue length of the routing node $i$ is greater than $60 \%$. When the ratio of the sending queue length of the routing node $i$ to the maximum queue length exceeds $80 \%$, the exponent is defined as $S Q_{i}=$ 2 ; otherwise, $S Q_{i}=0$. The received queue sat- uration level $R Q_{i}$ is also defined similarly for node $i$.

Because there is a delay between the calculation of the route and the use of the last route, it is better to have the ability to predict the congestion trend in the future. Therefore, we introduce the congestion prediction mechanism in the routing criterion calculation. The specific method is to do a comparison between the current saturation sum (including the receiving queue and the sending queue) and the previous statistical sum of the saturation. The pre-judgment factor $P_{i}$ and saturation difference threshold $\mu$ are defined as:

- The sum of the current saturation $>$ the sum of the previous statistics and the difference $>\mu$, then $P_{i}=1$.

- The sum of the current saturations $<$ the sum of the previous statistics and the difference $>\mu$, then $P_{i}=-1$.

- The sum of the current saturation and the saturation of the previous statistic is less than or equal to 0 , then $P_{i}=0$.

From the collected node data described above, it is possible to define an integrated link weight value $W_{i}$ between the node $i$ and its neighbor node. It is calculated using the following expression (1):

$$
W_{i}=\left\lfloor\frac{C_{i}}{B_{i}} \times 10\right\rfloor+S Q_{i}+R Q_{i}+P_{i}
$$

In order to make the link without the congestion node more advantageous than the link with the congestion node, an over-limit accumulation mechanism is adopted to improve the handling of routing in this special case. The specific method is that the weight value of a single node is calculated, and then a congestion threshold $n$ is set according to the specific circumstances of the network. When the weight value of a single node exceeds the congestion threshold, the node is considered to be congested; the degree of congestion is measured by the excess value. When the weight value is calculated, the excess is added to the final weight value $W_{i}$. The weight value of the improved node is calculated as follows:

$$
\begin{aligned}
& W_{i}=W_{i}+\left(W_{i}-n\right) \text { when } W_{i}>n \\
& W_{i}=W_{i} \text { when } W_{i} \leq n
\end{aligned}
$$


To sum up, the total weight $W_{p}$ of paths with $k$ nodes is the formula (3):

$$
W_{p}=\sum_{i=1}^{k}\left(\left\lfloor\frac{C_{i}}{B_{i}} \times 10\right\rfloor+S Q_{i}+R Q_{i}+P_{i}\right)
$$

\subsection{Load Balanced Multi-path Routing Mechanism}

The main idea of the LBDSRM algorithm is that after the route discovery process the sending task is distributed proportionally according to the total weight of the integrated link of each path, and the data is sent by using several optimal paths. Data is sent at the same time to each node real-time monitoring of its own state. Once there is a big change, the source node is in time notified to make the necessary adjustments. The algorithm also uses a back-off mechanism in a timely manner, so that the multi-path transmission task and the single-path transmission task have certain network fairness in the event of network resource contention. The bottleneck node congestion can be effectively avoided in this case. The dynamic load balancing of the network is achieved in the algorithm, and the utilization rate of the whole network resource is greatly improved.

Several parameters of the algorithm need be set. The optimal parameter values of the algorithm may be different in different network environments. Simulation of a network environment is taken as an example to show the best configuration, as described below:

In the route discovery process, the source node $S$ discovers that the destination node $D$ has $K$ effective paths, and the order of the weights is $P_{1}, P_{2}, \ldots, P_{K}$.

- When $K=1$, there is only one path, and the assignment ratio of the path $P_{1}$ is $100 \%$.

- When $K=2$, there are 2 paths, let $X=\left(W P_{2}-W P_{1}\right) / W P_{1}$

- When $X \leq 5 \%$, the task distribution ratio of $P_{1}$ and $P_{2}$ is $50 \%$.

- When $20 \%>X>5 \%, P_{1}$ task distribution ratio is $70 \%$, and $P_{2}$ task distribution ratio is $30 \%$.
- When $40 \%>X \geq 20 \%$, the task allocation ratio of $P_{1}$ is $90 \%$, and $P_{2}$ is $10 \%$.

- When $X \geq 40 \%$, the task allocation ratio of $P_{1}$ is $100 \%$, and $P_{2}$ is $0 \%$.

- When $K=3$, there are three paths, let $X=\left(W P_{2}-W P_{1}\right) / W P_{1}$ (first $P_{2}$ and $P_{3}$ as a whole).

- When $X \leq 5 \%$, the task allocation ratio of $P_{1}$ and $P_{2} P_{3}$ is $50 \%$.

- When $20 \%>X>5 \%, P_{1}$ task distribution ratio is $70 \%$, and $P_{2} P_{3}$ is $30 \%$.

- When $40 \%>X \geq 20 \%$, the task allocation ratio of $P_{1}$ is $90 \%$ and that of $P_{2} P_{3}$ is $10 \%$.

- When $X \geq 40 \%$, the task allocation ratio of $P_{1}$ is $100 \%$, and $P_{2} P_{3}$ is $0 \%$.

- $\quad$ For the task assignment of $P_{2}$ and $P_{3}$, the calculation is performed by referring to the algorithm when $K=2$.

- When $K>3$, the best first three paths are selected, and the distribution algorithm is calculated according to $K=3$.

In order to make sure that the nodes with only single-path routing have certain network fairness in transmitting data, the node initiates a backoff algorithm when the task of multipath transmission and the task of only single-path transmission are contention on some node or some link. If the node is currently $W$ $<n$ (congestion threshold), its weight is $W=$ $n$. Its change is notified to the source node of the sending task, and it is required to adjust the proportion of its sending task according to the new node state data. If the node current $W \geq n$ (congestion threshold), its weight $W=\infty$, the source node of the sending task is notified to avoid the assignment of the transmission task on this path, and the transmission proportion of other paths is adjusted.

To avoid generating excessive route responses during route discovery, the protocol also specifies the number of times - a route response will be generated. In the LBDSRM protocol, only three paths are used. There is no need to find too many paths in route discovery. One RREQ can only respond once to the intermediate node, but can respond to the first three arrivals for the destination node. 
Multi-path routing load balancing algorithm flow chart is shown in Figure 1.

\subsection{LBDSM Protocol Work Process}

\subsubsection{Route Discovery Process}

When a mobile node needs to send out information and the routing cache does not have the necessary routing information, the route discovery process is initiated and a RREQ is sent by using flooding. The RREQ structure includes the address of the source node, the address of the destination node, the unique sequence number, the accumulated link weights, and so on. Each node that receives RREQ first checks its own routing request table to see whether it needs to process the message:

- If the address of the node is already in the route record of the RREQ packet, the node discards the request packet and does not process it.

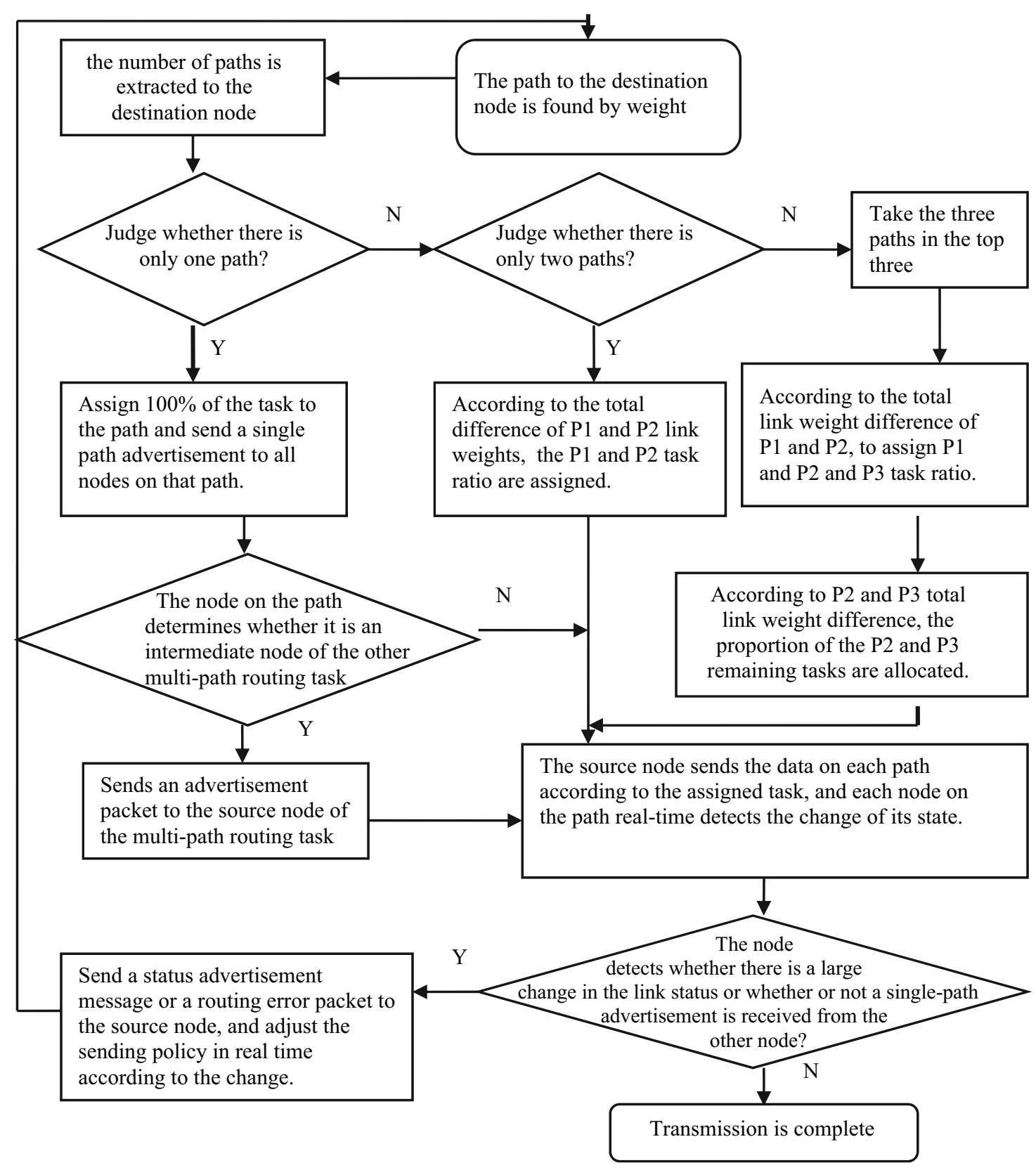

Figure 1. Multi-path routing load balancing algorithm flow chart. 
- If there is no source node and request sequence number of the RREQ message in the routing request table, the node adds its current real-time link weight value to the accumulated link weight item in the RREQ message, then checks whether it is the destination node in the RREQ packet. If yes, a route reply packet is sent to the source node; if not, its own address is added to the route record table of the route request message, then the extended route request message is forwarded to all the forward links.

- If the RREQ packet contains the source node and the request sequence number in the RREQ packet, this indicates that the node has processed the same RREQ packet recently. The node will check the destination node - whether it has reached the number of processing sessions. If reached, the request message is not received and request packet will be discarded; otherwise, a route reply packet is sent in the router to the source node.

A route request from the source node may receive multiple return route responses, and each route reply message carries valid path information to the destination node. Each path information includes not only a detailed route to the destination node, but also the cumulative link weight for this path. Unlike the DSR, the protocol records all the returned path information and the corresponding accumulated link weights, and multi-path routing is implemented at different aggregate link weights.

\subsubsection{Route Maintenance Process}

When multiple routing information is established, the route maintenance process is entered. The route maintenance process is mainly responsible for the following tasks:

1. The validity between real-time monitoring and the link of the neighbors: the response changes are caused in topology by the routing information changes. LBDSRM detects errors in link transmission through link detection, passive acknowledgment, and acknowledgment packet transmission.
In this case, the relevant node sends out a routing error packet. By sending "routing error packet messages and acknowledgments", the routing failure is reported to the mobile node, and the report should be promptly propagated to maintain the validity of the route after each routing node updates. Before the node is resumed, the network temporarily deletes the node from the cache of other nodes and deletes all transmission paths which are connected to the node.

2. Real-time monitoring of the node's link state: the node state change reporting mechanism is timely started, and dynamic load balance is achieved. When the node monitors its own real-time link status and the history records weight difference, these are recorded in the node weight record table. If the link notification threshold is reached, the current link status is compared with the original one. A routing node state is sent by the source node, where the advertisement message is changed to the source node, and the task proportion of the multi-path route of the source node is adjusted according to the content of the report. The multi-path routing mechanism is combined with the node change reporting mechanism, which can effectively reduce the probability of network load inequality.

The single-path route and the multi-path route link competition is real-time monitored in the nodes. When a node detects that it is the contention node of the single-path and multi-path routing tasks, the node modifies the $W$-value according to the real-time status of the node. A node-state change advertisement message is sent to the source node of the multi-path route, and the source node adjusts the transmission task proportion of the multiple paths according to the content of the report. In this way, the timely backoff mechanism is adopted, and the multi-path routing task is made to avoid the competition path in advance, so as to reduce the possibility of congestion occurrence. The overall performance of the system is improved and the purpose of load balance is achieved. 


\section{Protocol Simulation and Discussion}

\subsection{LBDSM Agreement Working Example}

As shown in Figure 2(a), when node $S$ data is sent to $D$, three paths to node $D$ (no other node contention) are found in the route discovery. The path state of the composite link weights are path $1=18$, path $2=24$, and path $3=15$, respectively. According to LBDSRM protocol, the $27 \%, 3 \%$ and $70 \%$ of the transmission task are assigned to the three paths by the multi-path routing algorithm. The main portion is placed on the best path 3 , while the data are sent simultaneously to three paths.

As is shown in Figure 2(b), after running for a period of time, node A needs to send data to node $B$. It only finds one path to the destination

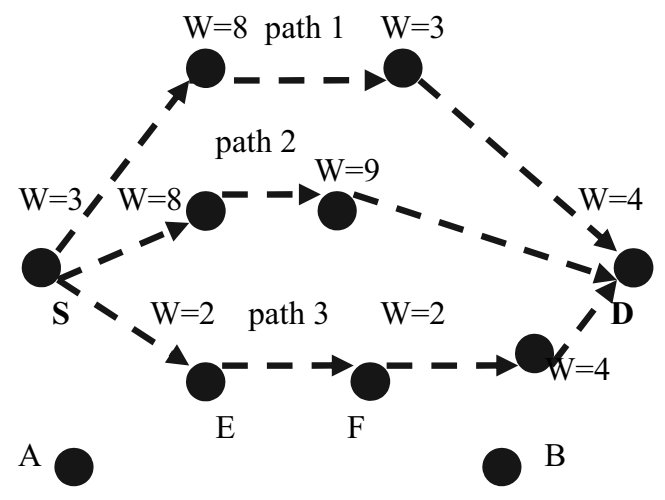

(a) Example $\mathrm{A} \rightarrow \mathrm{D}$

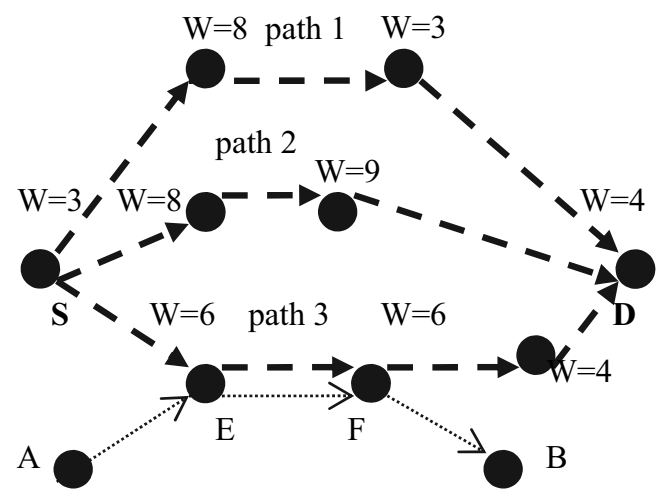

(b) Example $\mathrm{A} \rightarrow \mathrm{B}$

Figure 2. LIBDRM protocol specific example map. node (coinciding with path 3 which is used by $S$-node task). In order to make multi-path transmission, the node A sends an avoidance notice to the other nodes on the path, so that the state weights of the nodes $E$ and $F$ are changed from 2 to the congestion limit value 6 , and the node $S$ is informed. When the node $S$ has not yet completed sending the task, the new link state data will be used to send tasks, and the three routes are re-allocated. The integrated link weights are path $1=18$, path $2=24$, and path $3=23$ respectively for the new path state. According to LBDSRM protocol, $70 \%, 15 \%, 15 \%$ of the transmission task are allocated for three routes in the multi-path routing algorithm, and the main portion is on the path 1 . This effectively alleviates the sending task on the path 3 with the contention node. The single-path sending task is reserved to the node Also the congestion of the bottleneck node is avoided in this case, and the dynamic load balance of the network is realized.

\subsection{Simulation Environment and Parameter Setting}

In the simulation parameter selection, we use the system default settings, for example 802.11 basic data bandwidth which is 2 Mbits, in the choice of packet size. CBR business generator whose package size is 512 byte is used. The same simulation scenario is used in each routing protocol in which 50 nodes are randomly arranged in the $600 \mathrm{~m} \times 600 \mathrm{~m}$ network, and each node has the same node movement model at moving speed of $20 \mathrm{~m} / \mathrm{s}$ with random uniform distribution and pause simulation time $0 \mathrm{~s}$, $10 \mathrm{~s}, 20 \mathrm{~s}, 40 \mathrm{~s}, 100 \mathrm{~s}$. When the parameters of the improved routing protocol are configured, better setting parameters are selected according to the results of the test data. For example, the value of the congestion threshold $\mathrm{n}$ is set to 6 , the saturation difference threshold to 0.05 , the link change notification threshold to $f=0.2$. The rest of the parameters use the values described previously.

\subsection{Simulation Results and Analysis}

The LBDSM protocol, DSR protocol and MSR protocol are simulated in the NS2 environment, 
and the average end-to-end delay and normalized routing overhead data are obtained. The average end-to-end delay is the most important measure of the optimal load-balancing routing algorithm. The normalized route cost is defined by the number of the routing management packets. The destination node needs to receive at least one packet successfully. Normalized route cost is the main technical index to measure the efficiency of routing protocols. The numbers of source nodes in the simulation are: 10 and 30 respectively. Node pause time is: $0 \mathrm{~s}$ (very high mobility), 10 s, 20 s, 40 s, 100 s. Packet trans- mission rate is 4 packets/sec. The experimental data results are shown in Figure 3 and Figure 4, respectively.

From Figure 3, both the LBDSRM protocol and the MSR protocol use the multi-path routing mechanism, and they are better than the DSR protocol in the environment of 10 source nodes (light load) or 30 source nodes (overload). The average end-to-end delay is short, which means that the multi-path routing mechanism can effectively distribute the network load and avoid node congestion, and the network load balance
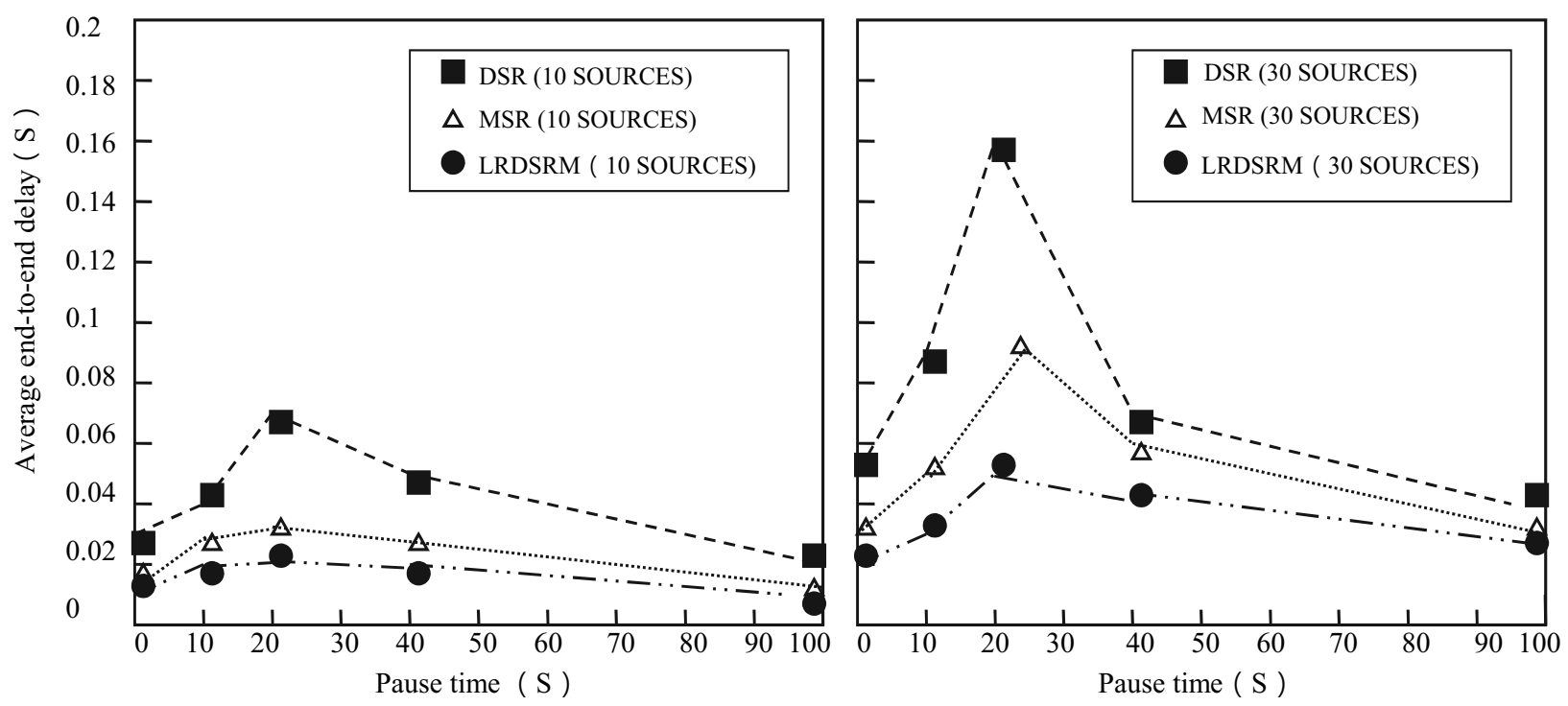

Figure 3. Average end-to-end delay comparison graph

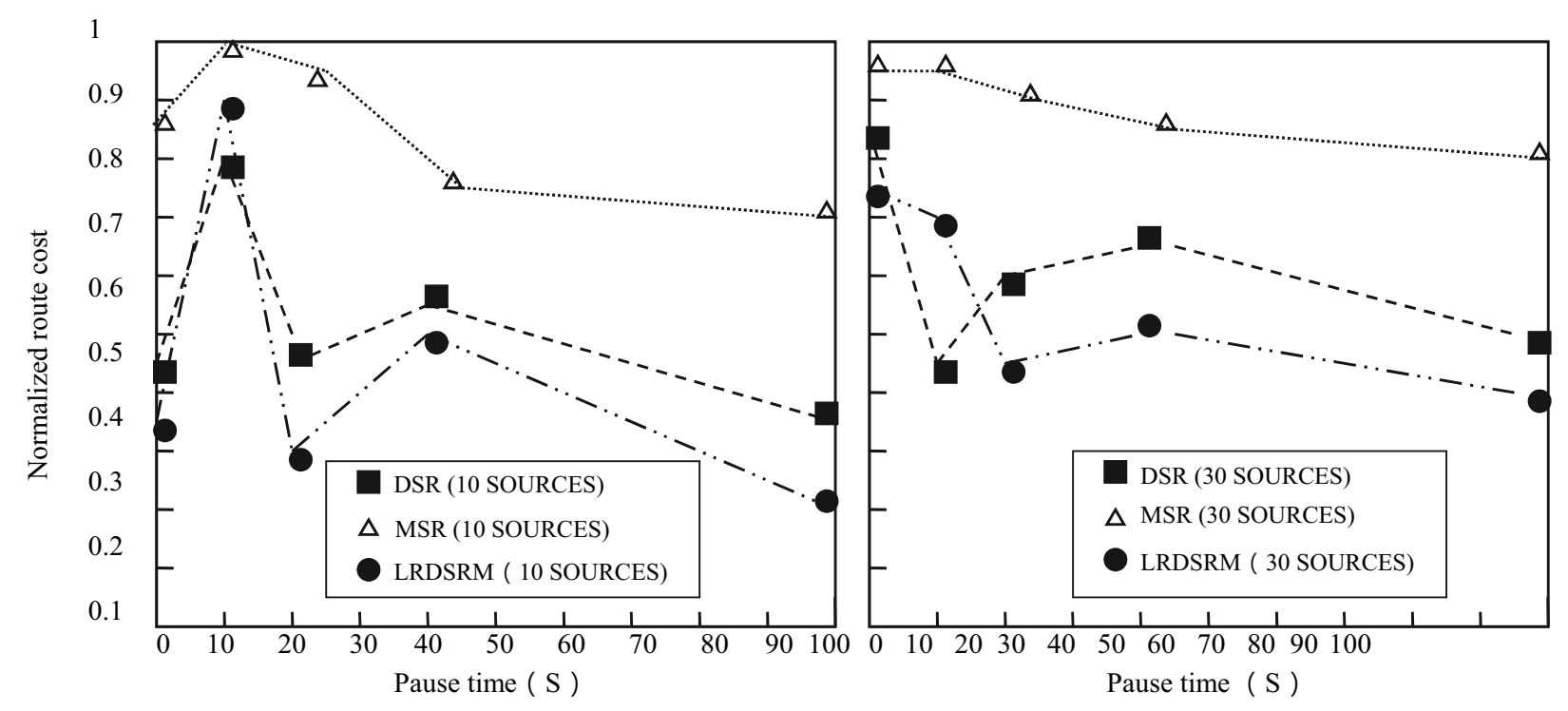

Figure 4. Normalized route cost comparison graph. 
is realized. The end-to-end delay of the LBDSRM protocol is much lower than that of the MSR protocol, especially in the case of 30 source nodes. This is mainly because the LBDSRM protocol takes into account the link state and node queue saturation degree, and the predictive factor and overrun accumulation mechanism are introduced. The link back-off strategy is solved for the single-path routing tasks and multi-path routing tasks, so it is more effective than the MSR protocol to avoid the actual congestion. The higher dynamic load balancing effect is achieved. It can effectively avoid the delay increase which is usually caused by packet loss, queue buffering and retransmission due to node overload. As the residence time increases, the end-to-end delay of these protocols decreases. The mobility of nodes decreases after the increase of the dwell time, which reduces the cost of routing maintenance.

As can be seen from Figure 4, MSR protocol overhead is much larger than in case of the LBDSRM and the DSR protocols. This is mainly because the MSR protocol measures the link quality by measuring the round-trip time of a unicast packet. This means that a large number of probe packets need to be sent, so the overhead is kept at a high level. The LBDSRM protocol and DSR protocol do not need to send additional probe packets, so the overhead is small. Generally speaking, the LBDSRM protocol is advantageous over the DSR protocol, because the LBDSRM protocol adopts the multi-path mechanism, and reduces the probability of re-initiating the route discovery due to the interruption of the link. Therefore, the overhead is relatively small. However, when the network changes rapidly, the change notification mechanism in the LBDSRM protocol can cause more change notification messages to be sent. In this special case, the overhead may be higher than that of the DSR protocol. Figure 4 also confirms this point.

\subsection{Comparison with Other Works}

Besides DSR and MSR, there are many other methods developed for the same situation. They usually do not have the strong substantiation for their scalability and fault-tolerance as they are studied with only a few thousand nodes and without considering failures or realistic loads. Specifically, for the decentralized scheduling methods PD MinRC [27], iHLBA [28], GA [29], QAFT [30] and New Model [31], the experiments are conducted with less than 10000 nodes, without considering node failures or realistic workloads. Table 1 compares our work with some of these works.

\section{Conclusion}

There are two basic work modes of multi-paths routing:

1. Multiple paths (simultaneous multi-path) are used at the same time, and

2. The main path is used first; replacement paths (replace the multi-path) are used after the main path failed.

When it comes to deal with the load balancing problem, the multi-path mode is superior to the replacement multi-path mode. Therefore, to some extent, many existing multi-path protocols are not desirable. For example, the disadvantage of MSR is that the processing overhead increases drastically when sending packets. The SMR has too many RREQ packets to transmit.

Table 1. Comparison with other works.

\begin{tabular}{|c||c|c|c|c|}
\hline Work Name & Number of Nodes & Failure & LB & Work load \\
\hline \hline LBDSRM & 1000000 & Yes & Yes & Traces \\
\hline PD_MinRC & 500 & Yes & Yes & Poisson Distribution \\
\hline iHLBA & 100 & No & Yes & Random Generation \\
\hline GA & 5 & No & Yes & Random Generation \\
\hline QAFT & 256 & Yes & No & Uniform Distribution \\
\hline New Model & 1000000 & Yes & No & Traces \\
\hline
\end{tabular}


In AOMDV, the data transmission often cannot use the shortest path. The load balancing mechanism of the shortest path routing algorithm has the problem of network congestion due to excessive load on some nodes. The widespread deployment and application requirements of the wireless network are increasing rapidly. There is a large space for further optimization of multi-path routing protocols and further improvement of equilibrium mechanisms.

A multi-path routing protocol is proposed in this paper with high load balancing capability. Firstly, existing wireless WMN routing metrics and multi-path routing protocols are reviewed to outline the balance problem. Then, based on the DSR protocol and multi-path routing, a load balancing dynamic source routing protocol LBDSRM is proposed. The protocol adopts the comprehensive link-state routing criterion and multi-path information to solve the problem of dynamic load balance in the network. The full utilization of network resources can be realized. Simulations of the LBDSRM protocol, MSR protocol and DSR protocol are carried out in the environment of network simulation tool NS-2. The experimental results show that the LBDS$\mathrm{RM}$ protocol is more advantageous, especially in the treatment of load balancing problem. The LBDSRM protocol is useful in the heavy network load environment that can be expected in the future wireless networks.

\section{Acknowledgement}

This work has been sponsored by 2018 Hunan Foreign Economic Research Institute Scientific Research Project "Personal Physiological Monitoring Information Algorithm Based on Portable Wearable Devic", 2018 Hunan Provincial Department of Education Scientific Research Project "Design and Research of Intelligent Physiological Monitoring System Based on Wearable Portable Device", Changsha, China.

\section{References}

[1] K. T. Chai, et al., "Load Balanced Routing Protocols for Ad hoc Mobile Wireless Networks", IEEE Communications Magazine: Articles, News, and Events of Interest to Communications Engineers, vol. 8, no. 8, pp. 78-84, 2009.

http://dx.doi.org/10.1109/MCOM.2009.5181896

[2] C. E. Perkins and P. Bhagwat, "Highly Dynamic Destination-Sequenced Distance-Vector (DSDV) Routing for Mobile Computers", in Proc. of UK:ACM SIGCOMM'94, 1994, pp. 234-244.

[3] S. Murthy and J. J. Garcia-Luna-Aceves, "An Efficient Routing Protocol for Wireless Networks", Mobile Networks and Applications, vol. 1, no. 2, pp. 183-197, 1996.

http://dx.doi.org/10.1007/BF01193336

[4] X. Chen, H. Peng, et al., "A Multi-channel MAC Protocol for Wireless Sensor Networks", in Proc. of The Sixth IEEE Int. Conference on Computer and Information Technology (CIT'06), 2006. http://dx.doi.org/10.1109/CIT. 2006.14

[5] J. Su Jing and W. Guo, "Summarization of Wireless Mobile Ad hoc Network Routing Protocols", China Measurement Technology, vol. 35, no. 2, pp. 91-93, 2005.

http://dx.doi.org/10.3969/j.issn.1674-5124.2005. 02.034

[6] C. Perkins et al., "Ad hoc on Demand Distance Vector (AODV) Routing", in Proc. of The Internet Engineering Task Force (IETF), 2003. http://dx.doi.org/10.17487/RFC3561

[7] D. Johnson et al., "The Dynamic Source Routing Protocol (DSR) for Mobile Ad Hoc Networks for IPv4", in Proc. of RFC 4728, 2007.

http://tools.ietf.org/html/rfc4728

[8] V. Park and M. S. Corson, "Temporally-Ordered Routing Algorithm (TORA) Version 1 Functional Specification", Internet-Draft, IETF, Draft-IetfManet-Tora-Spec-04.txt, July 2001.

[9] Z. J. HAAS, "A New Routing Protocol for the Reconfigurable Wireless Networks", in Proc. of USA:IEEE ICUPC'97, 1997, pp. 562-566.

[10] S. J. Lee and M. Gerla, "Split Multi-path Routing with Maximally Disjoint Paths in Ad Hoc Network", in Proc. of IEEE Int. Conference on Communications, 2001, pp. 3201-3205.

[11] M. K. Marina and S. R. Das, "On-Demand Multipath Distance Vector Routing for Ad Hoc Network", in Proc. of IEEE ICNP, 2001. http://dx.doi.org/10.1109/ICNP.2001.992756

[12] Y. T. Shu et al., "Multi-path Source Routing in Wireless Ad Hoc Networks", Acta electronica sinica, vol. 30, no. 2, pp. 279-282, 2002. http://dx.doi.org/10.3321/j.issn:0372-2112.2002. 02.034

[13] S. J. Lee and M. Gerla, "AODV-BR Backup Routing in Ad Hoc Networks", in Proc. of IEEE WCNC, 2000, pp. 1311-1316. http://dx.doi.org/10.1109/WCNC.2000.904822

[14] S. Lei and G. Lindong, "A Node Disjoint Multipath Routing Protocol in Mobile Ad Hoc Net- 
works", Computer engineering and applications, vol. 41, no. 3, pp. 159-161, 2005. http://dx.doi.org/10.3321/j.issn:1002-8331.2005. 03.051

[15] P. Antoniou and A. Pitsillides, "A Bio-inspired Approach for Streaming Applications in Wireless Sensor Networks Based on the Lotka-Volterra com-petition Model". Computer Communications, vol. 33, no. 17, pp. 2039-2047, 2010. http://dx.doi.org/10.1016/j.comcom.2010.07.020

[16] C. J. Lin and C. F. Chou, "Route-Aware Load-Balanced Resource Allocation for Wireless Mesh Networks", in Proc. of IEEE Int. Conference on Wireless Communications and Networking (WCNC'07), 2007.

http://dx.doi.org/10.1109/WCNC.2007.572

[17] S. Waharte et al., "Interference-Aware Routing Metric for Improved Load Balancing in Wireless Mesh Network", in Proc. of IEEE Int. Conference on Communications, 2008.

http://dx.doi.org/10.1109/ICC.2008.561

[18] N. M. Kharasani and Z. A. Zukarnain, "Performance Evaluation of Routing with Load-balancing in Multi-radio Wireless Mesh Networks", International Journal of Digital Content Technology and Its Applications, vol. 5, no. 2, pp. 64-71, 2011.

[19] W. J. Feng et al., "Multi-path and Load Balancing Routing Protocol in Multi-radio Wireless Mesh Network", Journal of System Simulation, vol. 23, no. 11 , pp. 2518-2522, 2011.

[20] C. X. Liu, et al., "A Load Balanced Routing Protocol Based on Ant Colony Algorithm for Wireless Mesh Network", in Proc. of IEEE Int. Conference on Genetic and Evolutionary computing (ICGEC 2011), 2011, pp. 295-298. http://dx.doi.org/10.1109/ ICGEC.2011.73

[21] A. Raniwala and T. Chiueh, "Architecture and Algorithms for an IEEE 802.11-based Multi-channel Wireless Mesh Network", in Proc. of Int. Conference on the IEEE Computer and Communications Societies (INFOCOM'05), 2005, pp. 2223-2234. http://dx.doi.org/10.1109/INFCOM.2005.1498497

[22] I. Motorola et al., "Simple Efficient Extensible Mesh (SEE-Mesh) Proposal", IEEE Document Number: 11-05/0562r1, 2005.

[23] Y. B. Liu et al., "Load Balancing Routing Protocol Based on Traffic Prediction for Wireless Mesh Networks", Computer Science, vol. 44, no. 1, pp. 78-84, 2017.

http://dx.doi.org/10.11896/j.issn.1002-137X. 2017.01.021

[24] Y. C. Zhang et al., "Sandpile Model Based Load Balancing Algorithm in Wireless Mesh Networks", Computer Science, vol. 45, no. 8, pp. 84-87, 2018.

http://dx.doi.org/10.11896/j.issn.1002-137X. 2018.08.015
[25] R. Draves et al., "Routing in Multi-Radio, MultiHop Wireless Mesh Networks", in Proc. of ACM MobiCom, Philadelphia, PA, 2004, pp. 114-128.

[26] L. Ma and M. K. Denko, "A Routing Metric for Load Balancing in Wireless Mesh Networks", in Proc. of IEEE 21st Int. Conference on Advanced Information Networking and Applications Workshops (AINAW'07), 2007.

http://dx.doi.org/10.1109/AINAW. 2007.50

[27] B. Jasma and N. Raju, "Performance-driven Load Balancing with Primary-backup Approach for Computational Grids with Low Communication Cost and Replication Cost", IEEE Transactions on Computers, vol. 62, no. 5, pp. 990-1003, 2013. http://dx.doi.org/ 10.1109/ TC.2012.44

[28] Y. H. Lee et al., "Improving Job Scheduling A1gorithms in a Grid Environment", Future Generation Computer Systems, vol. 27, no. 8, pp. 991-998, 2011. https://doi.org/10.1016/j.future.2011.05.014

[29] Y. J. Li et al., "A Hybrid Load Balancing Strategy of Sequential Tasks for Grid Computing Environments", Future Generation Computer Systems, vol. 25 , no. 8, pp. 819-828, 2009. https://doi.org/10.1016/j.future.2009.02.001

[30] R. Z. Khan and M. F. Ali, "An Efficient Diffusion Load Balancing Algorithm in Distributed System", International Journal of Information Technology and Computer Science (IJITCS), vol. 6, no. 8, pp. 65-71, 2014. http://dx.doi.org/10.5815/ijitcs.2014.08.09

[31] J. Celaya and U. Arronategui, "A Task Routing Approach to Large-Scale Scheduling", Future Generation Computer Systems, vol. 29, no. 5, pp. 1097-1111, 2013. https://doi.org/10.1016/j.future.2012.12.009
Received: July 2018 Revised: February 2019 Accepted: February 2019 


\section{Contact addresses:}

Lu Yan

Hunan International Economics University

Changsha, Hunan Province

P. R. China

e-mail: 66754252@qq.com

Ding Xiong*

Hunan International Economics University

Changsha, Hunan Province

P. R. China

e-mail: matlab_bysj@126.com

*Corresponding author

Lu YAN received the bachelor's degree in Computer Science and Technology from Hunan Normal University, China, in 2002. She received her Master's degree in Technical Economics and Management from Central South University, China, in 2010. She is currently working as Senior Engineer at the Information Science and Technology, Hunan International Economics University. Her research interests include algorithm applications and distributed computing.

DING XIONG received the bachelor's degree in Computer Science and Technology from Hunan University of Science and Technology, China, in 2003. He received his Master's degree in Computer Science and Technology from Hunan University, China, in 2010. He is currently working as Senior Engineer at the Information Science and Technology, Hunan International Economics University. His research interests include network computing and algorithm applications. 